\title{
Validation of a 3D Pretreatment Quality Assurance Tool for Volumetric Modulated Arc Therapy (VMAT)
}

\author{
C. Francis Djoumessi Zamo ${ }^{1,2}$, M. Ndontchueng Moyo \\ ${ }^{1}$ GCS Radiothérapie, CH Angoulême, Saint-Michel, France \\ ${ }^{2}$ Centre for Atomic, Molecular Physics and Quantum Optics, Faculty of Science, University of Douala, Douala, Cameroon \\ Email: djoumess@gmail.com
}

How to cite this paper: Djoumessi Zamo, C.F. and Ndontchueng Moyo, M. (2021) Validation of a 3D Pretreatment Quality Assurance Tool for Volumetric Modulated Arc Therapy (VMAT). Open Access Library Journal, 8: e7434.

https://doi.org/10.4236/oalib.1107434

Received: April 19, 2021

Accepted: June 25, 2021

Published: June 28, 2021

Copyright $\odot 2021$ by author(s) and Open Access Library Inc.

This work is licensed under the Creative Commons Attribution International License (CC BY 4.0).

http://creativecommons.org/licenses/by/4.0/

\begin{abstract}
Implementation of a pretreatment quality assurance (QA) system needs a strong validation process and a good comprehension of the tool. The aim of this study is to validate COMPASS (IBA Dosimetry, Germany) as our 3D pretreatment QA tool for system of Pinnacle as treatment planning system and an ELEKTA synergy linear accelerator. Validation of the systems was performed with static and dynamic plans on AAPM TG-119 phantom and 10 real VMAT plans for prostate and head and neck. Comparison between point dose from TPS and COMPASS was performed to evaluate the confidence limit in high dose region (98.5\% and 98.6\%) and low dose region $(94.1 \%$ and 95.2\%) for COMPASS computed and reconstructed dose respectively. For planar dose the confidence limit was respectively $95.37 \%$ and $96.35 \%$ for COMPASS computed and reconstructed dose. Clinical validation was evaluated by comparing dose-volume parameters for real VMAT plans with TPS values (mean differences were below of $1 \%$ for the target). The comparison between 2D dose distribution from TPS and dose extracted from COMPASS computed and reconstructed for real VMAT plans were also performed (mean global gamma passing rate better than $94 \%$ and $98 \%$ for the $2 \% / 2 \mathrm{~mm}$ and $3 \% / 3 \mathrm{~mm}$ criteria). The $3 \mathrm{D}$ dose distribution comparison between TPS and COMPASS was also performed with good gamma score for global and local analysis. COMPASS was successfully evaluated as our 3D pretreatment system.
\end{abstract}

\section{Subject Areas}

Radiology

\section{Keywords}

COMPASS, VMAT, Pretreatment 


\section{Introduction}

Intensity-Modulated Radiation Therapy (IMRT) has proven successfully in improving the distribution of dose in patients. Its ability to improve coverage of the tumor while minimizing the dose to healthy organs results in high dose gradient. Volumetric Modulated Arc Therapy (VMAT) is the dynamic mode of IMRT delivery in which the leaves of the multileaf collimator (MLC) move continuously at independent speeds while the gantry rotates around the patient while the beam continuous on at different dose rate [1]. Since the conception of IMRT, many patient pretreatment quality assurance $(\mathrm{QA})$ techniques/tools have been proposed. These include ion chamber, film dosimetry, ion chamber array, diode array and EPID [2] [3] [4] [5] and have shown good utility as patient QA devices. Each of them has its limitations regardless of the kind of information we want to extract. Furthermore, these devices only allow the possibility to make a $2 \mathrm{D}$ analysis of measurements. The results from $2 \mathrm{D}$ analysis cannot be directly used to see the effects of the dose calculation and treatment delivery errors on the tumor dose or dose to the normal tissues inside the patient.

An ideal QA tool should be easy to implement, fast relevant and provide 3D information. The need for 3D QA is more so in today's era of VMAT because of the complexity of the rotational treatment technique that require the linac to dynamically control various parameters during the treatment delivery. Compass (IBA dosimetry, Germany) a new 3D patient QA device has recently been introduced by IBA. COMPASS has the potential to meet most of these requirements. The work of Boggula et al. [6] validated the use of COMPASS as a QA tool for VMAT. Swamy et al. [7] and Clemente-Gutiérrez et al. [8] have validated COMPASS respectively with Eclipse and Monaco treatment planning systems (TPS). However, no study to the best of our knowledge conducted a validation for Pinnacle TPS and Elekta treatment machine. The goal of the present work is to commission our COMPASS QA device and validate it as our 3D pretreatment QA system using Pinnacle TPS.

\section{Materials and Methods}

\subsection{The Treatment Unit}

The commissioning and all measurements were performed using a $6 \mathrm{MV}$ photon beam of our dual energy Synergy (Elekta, Stockholm, Sweden) machine. The system is equipped with an Agility multileaf collimator. The 160 leaves of $0.5 \mathrm{~cm}$ width projected at the isocenter.

\subsection{The Treatment Planning System}

The treatment planning system used is Pinnacle ${ }^{3}$ (Philips Radiation Oncology Systems, Fitchburg, WI), version 9.8. Beam profiles and depth doses were measured with the semiflex $0.125 \mathrm{cc}$ ionization chamber and the diamond detectors (PTW, Freiburg, Germany) for square field ranging from $1 \times 1$ to $40 \times 40 \mathrm{~cm}^{2}$. Output factors were measured using the diamond detectors (for small fields) and 
the PTW $0.125 \mathrm{cc}$ ionization chambers at $10 \mathrm{~cm}$ depth in a 3D water tank. The beam model created in Pinnacle and validated with the criteria based on the work of Starkschall et al. [9] and the Agility setting parameters were based on previous publications [10] [11] [12].

\subsection{The COMPASS System}

The COMPASS QA system (IBA Dosimetry, Schwarzenbruck, Germany) used for patient pretreatment quality assurance consists of:

- The COMPASS software (V3.1b) used for dose computation and analysis.

- A 2D array detector (MatriXX) with a gantry angle sensor for data collection and dose reconstruction.

The main function is to reconstruct the dose on patient CT based on the measurements taken with the $2 \mathrm{D}$ array detectors.

\subsubsection{COMPASS}

The dose computation in the COMPASS QA system is done by an independent dose calculation engine in order to double check the calculated dose by the TPS. The beam data in compass consists of depth dose, cross profiles curves and output factors obtained at SSD $100 \mathrm{~cm}$ (Beam model). Some mechanical and dosimetric characteristics of the linear accelerator for the given energy are also needed for fine-tuned the model. A collapsed cone convolution/superposition dose engine is implemented in COMPASS for calculating 3D dose distribution.

\subsubsection{MatriXX}

The 2D array detector (MatriXX) consists of a 1020 parallel plane ion chambers of $0.125 \mathrm{cc}$ with an active area of $24.4 \times 24.4 \mathrm{~cm}^{2}$ and a resolution of $7.619 \mathrm{~mm}$ at isocenter $100 \mathrm{~cm}$. The detector is mounted on the treatment unit head and rotates with the gantry. A build up layer of $5.0 \mathrm{~cm}$ is placed on the device for the measurements. The source detector distance is $76.2 \mathrm{~cm}$.

Beam model is based on the measure data from linac and its geometricals characteristics. To compute its dose, COMPASS used the RTPLAN (from TPS) and beam model. For reconstructed dose, the irradiated plan measure with MatriXX is computed with the beam model and the CT data as reconstructed dose. Both of them are compared to the RT dose from the TPS.

\subsection{The Validation Process}

The phantom used in our study is a scan of a set of water equivalent slabs of 20 $\mathrm{cm}$ thick. Plans were computed for different doses using Pinnacle TPS and were transferred to COMPASS for dose calculation. Similarly, the measured dose profiles were transferred to COMPASS reconstruction after measurements under a linear accelerator. Irregular's fields shape was investigated. TG-119 test plans as well as real patients were also used in the validation process.

\subsubsection{Special Fields}

Two special fields were chosen for validation of the beam modeling in the treat- 
ment planning system (TPS) and COMPASS QA system: a bar pattern and C shape beams (See Figure 1(a) and Figure 1(b)). Profiles at in a plan at $10 \mathrm{~cm}$ depth were performed COMPASS computed and reconstructed dose. The bar pattern consisted of an alternating opened and closed regions of $2 \mathrm{~cm}$ height, formed by the MLC leaves in a $10 \mathrm{~cm} \times 20 \mathrm{~cm}$ collimated field. $\mathrm{C}$ shape fields consisted of MLC leaves arrangements in the form of C letter. These special fields were irradiated with $800 \mathrm{MUs}$ and measure for COMPASS computed and reconstructed doses.

\subsubsection{TG-119 Test Plans}

The TG-119's phantom were downloaded as DICOM-RT data and planned following the guidelines of AAPM TG-119 [13] [14]. Dynamic plans were calculated in TPS and measured with a $0.125 \mathrm{cc}$ ionization chamber and MatriXX for COMPASS dose. The computed and reconstructed doses were compared to the planned dose in terms of point dose and gamma in different planar dose distributions ( $3 \% / 3 \mathrm{~mm}$, global normalization and $20 \%$ of dose threshold).

For point dose measurements with chamber, in order to take into account the effects of the couch attenuation, the daily linac output variations and the differences between the phantom and liquid water, we irradiated the phantom with two opposite parallel $10 \times 10 \mathrm{~cm}^{2}$ fields arranged isocentrically. The ratio of measured to planned doses were used to correct the others chamber measurements.

\subsubsection{Validation with Real Patient Plans}

A total of ten patients' (prostate and head and neck patients) VMAT plans were generated in order to compare the COMPASS compute and reconstruct dose. The patients contain three levels of dose for each planning target volume (PTV). The treatments consisted of a single arc in simultaneous integrated boost. The final dose calculation was performed using a $2 \mathrm{~mm}$ grid resolution and an adaptive

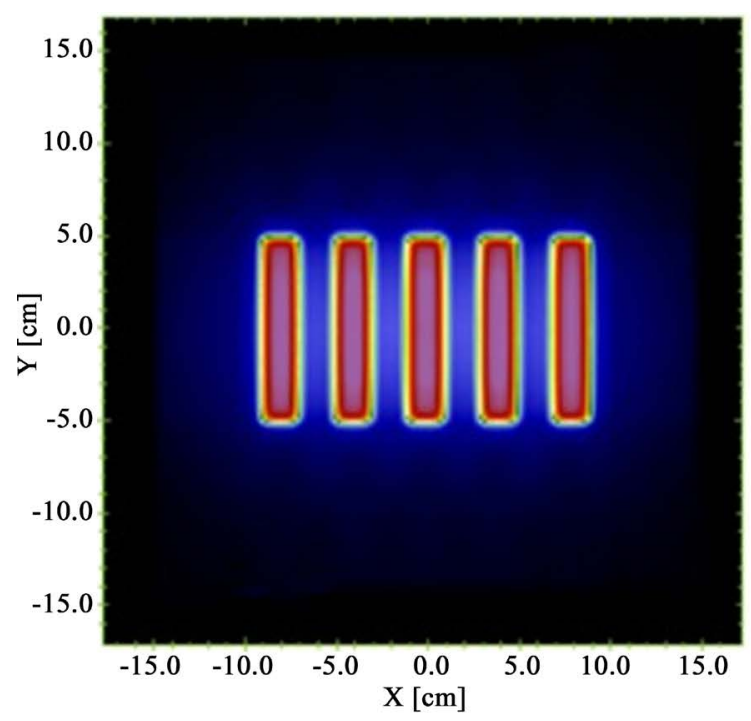

(a)

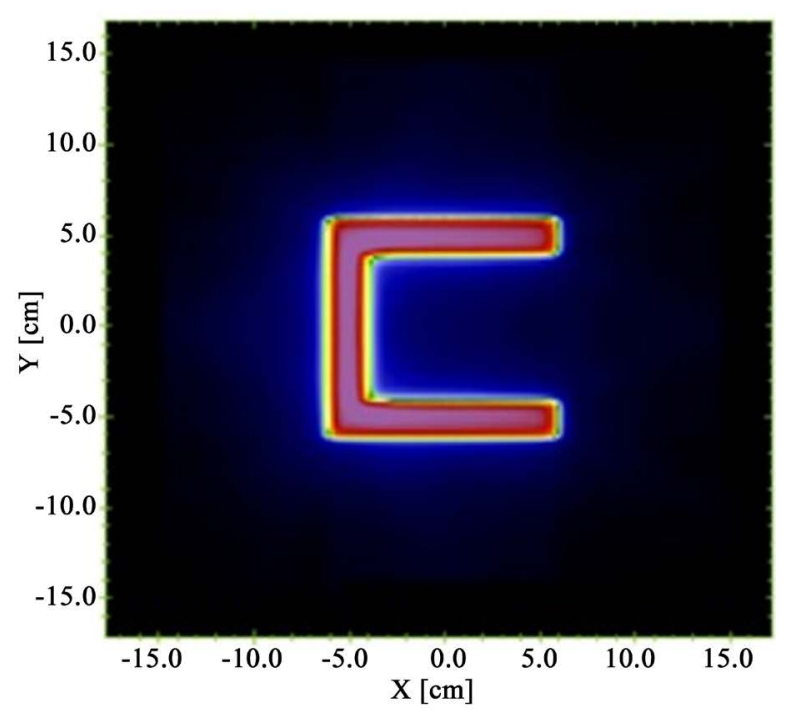

(b)

Figure 1. Special fields to test beam modeling in COMPASS and TPS: (a) bar pattern, (b) C-shape field. 
convolution algorithm. Dose level on ICRU point dose, gamma analysis (2\%/ $2 \mathrm{~mm}$ and $3 \% / 3 \mathrm{~mm}$, 3D local, 2D local and global normalization to maximum with a low dose threshold at $10 \%)$. Axial, coronal and sagittal analyses were performed.

\subsubsection{Plans with Intentionally Errors}

Intentional errors (gantry and collimator angle, MUs and MLC positions) were inserted in one $\mathrm{H} \& \mathrm{~N}$ plan and in one prostate plan. The plans without errors were used as reference in COMPASS. Dose differences at D95, tolerance dose at some organ at risk (OAR)'s and 3D average gamma were evaluated. A 2D gamma analysis was performed to compare the TPS to COMPASS's doses. The compute and reconstruct dose were compared to the planned dose in terms of DVH, point dose and gamma in different planes. Planar dose distributions were analyzed using gamma criteria $2 \% / 2 \mathrm{~mm}$ and $3 \% / 3 \mathrm{~mm}$ for both compute and reconstruct dose. The concept of "confidence limit" was used to describe the agreement between COMPASS (computed and reconstructed) and the TPS for planar dose and point dose.

\section{Results}

\subsection{Special Fields}

Dose profiles at depth $10 \mathrm{~cm}$ are plotted to compare TPS against COMPASS for the tests fields are presented in Figures 2-5.

With the aid of the 2D analysis tool from COMPASS, profiles through the central axis in the $\mathrm{x}$ and $\mathrm{y}$-axis were extracted. These tests were mainly used to validate tongue-and-groove leakage width parameter, MLC transmission, primary and scatter source characteristics and small fields dosimetry according to the work of Cadman et al. [15].

\subsection{The TG-119 Test Plans}

The TG-119 cases were planned with dynamic arcs and measured with MatriXX

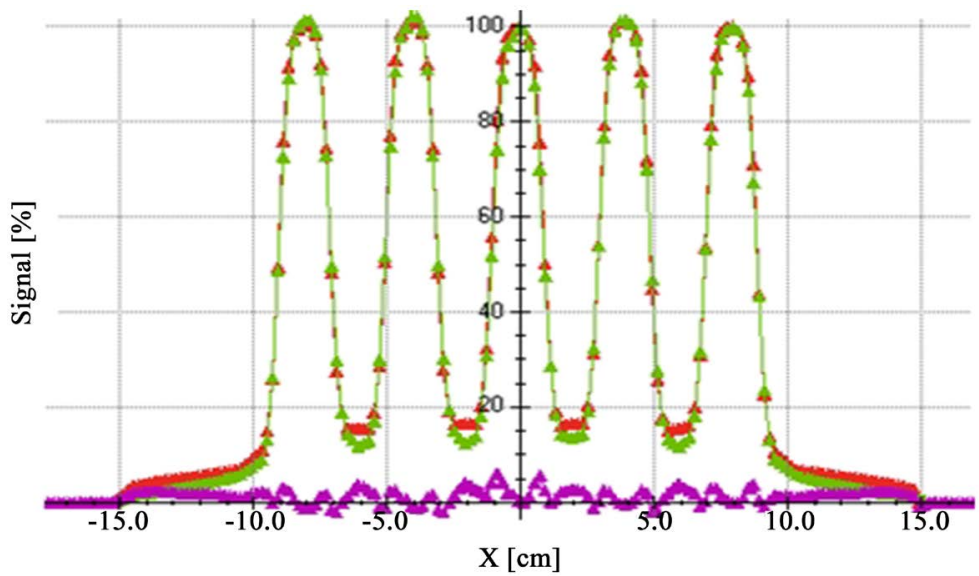

Figure 2. Bar pattern test $\mathrm{x}$-axis profiles reconstructed by COMPASS (green) and calculated by TPS (red) and the dose difference (purple). 


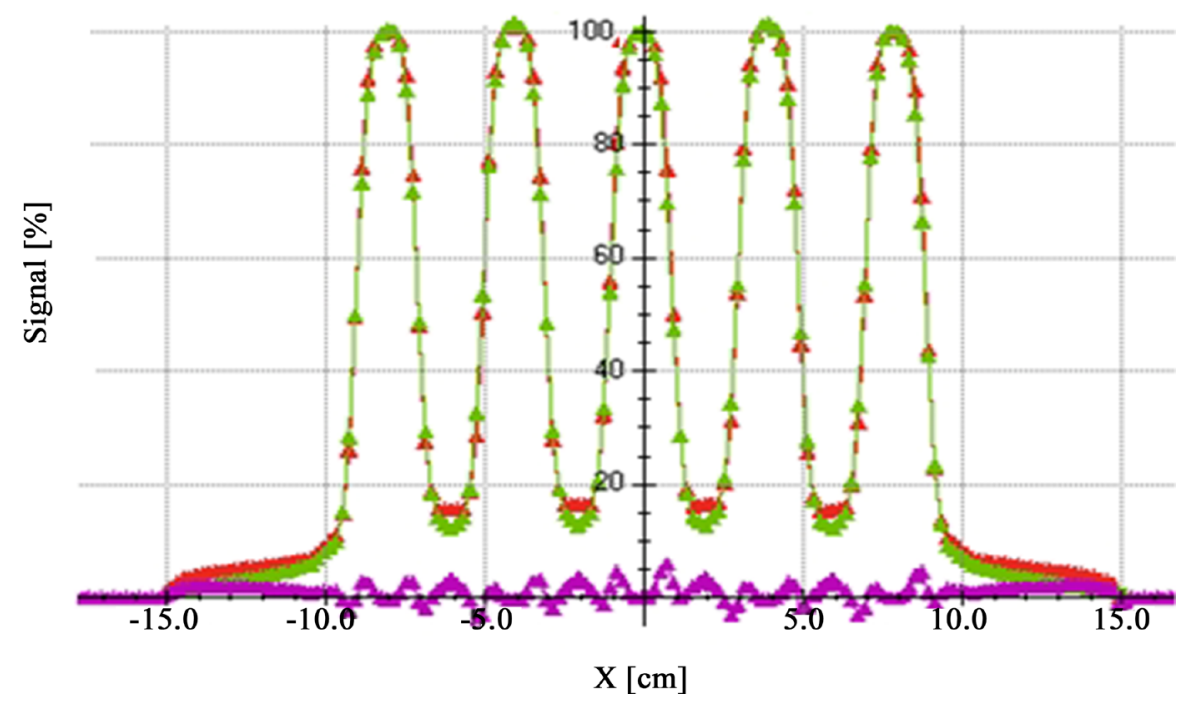

Figure 3. Bar pattern test $\mathrm{x}$-axis profiles computed by COMPASS (green) and calculated by TPS (red) and the dose difference (purple).

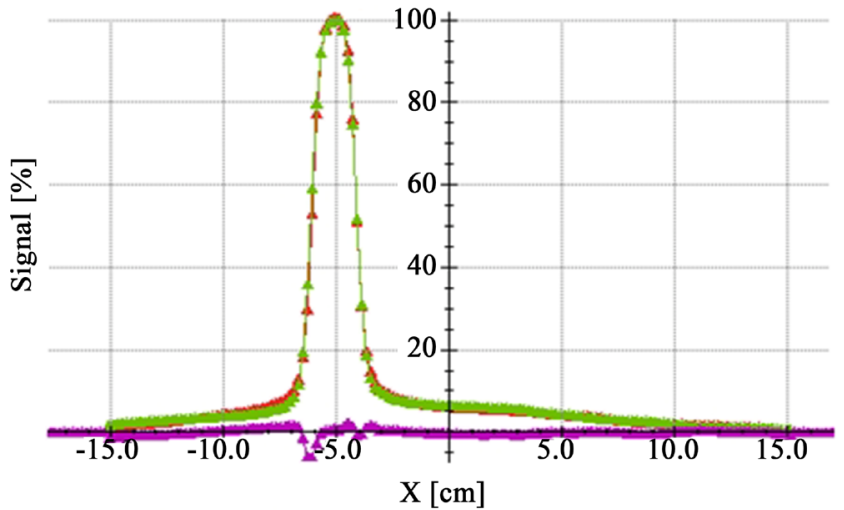

(a)

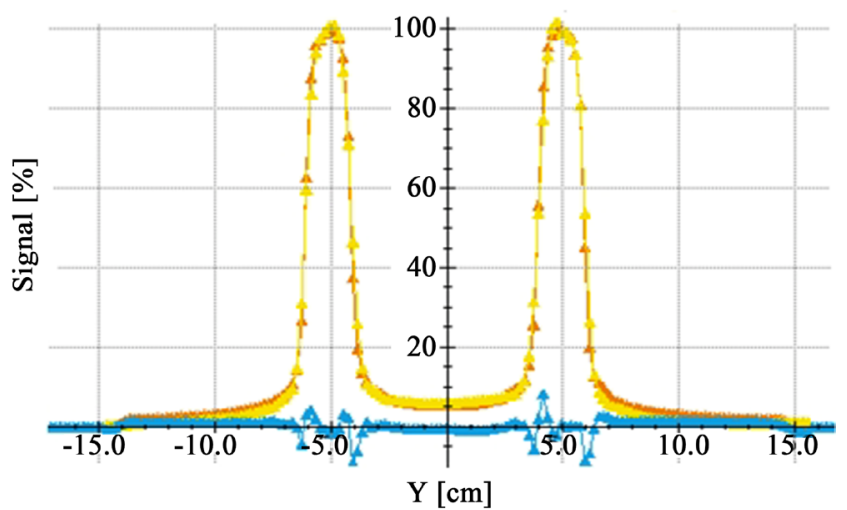

(b)

Figure 4. C-shape dose profiles comparison at $10 \mathrm{~cm}$ depth between COMPASS reconstructed and TPS; (a) X-axis, the red curve represents the TPS profiles, the green is from COMPASS and the purple is the dose difference. (b) Y-axis profile, the orange curve stands for TPS, the yellow for COMPASS and the blue is the dose difference.

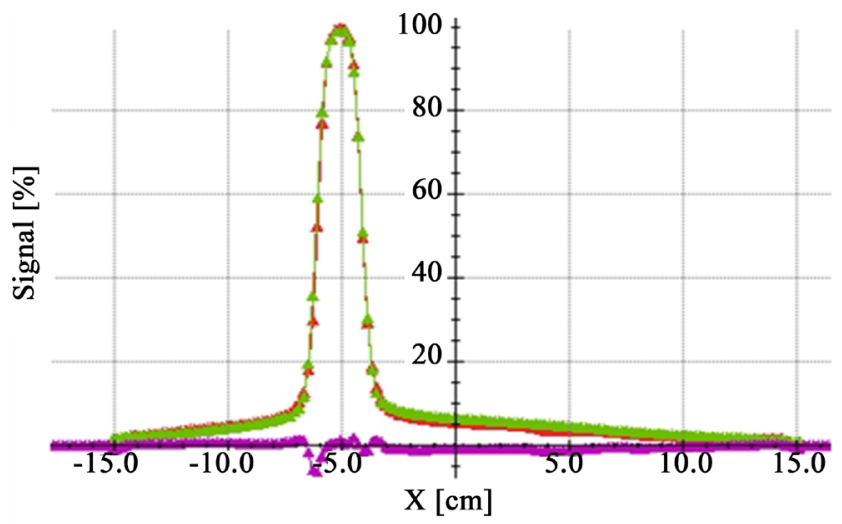

(a)

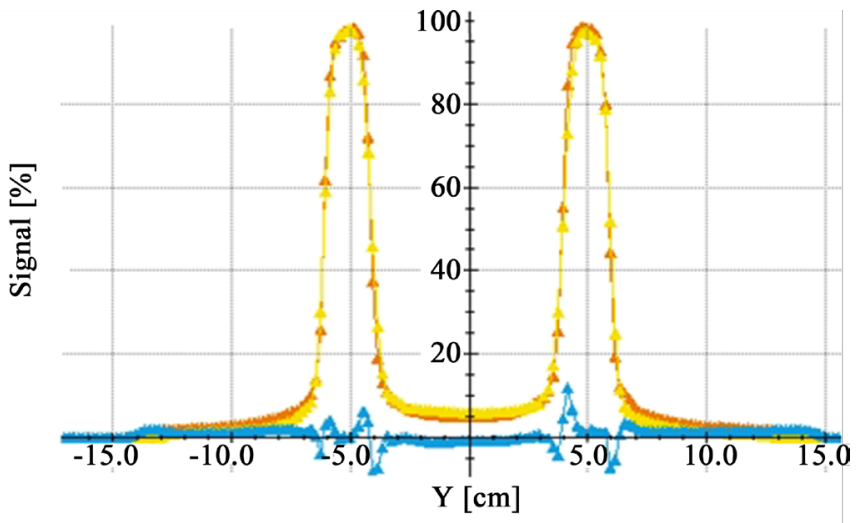

(b)

Figure 5. C-shape dose profiles comparison at $10 \mathrm{~cm}$ depth between COMPASS computed and TPS; (a) X-axis, the red curve represents the TPS profiles, the green is from COMPASS and the purple is the dose difference. (b) Y-axis profile, the orange curve stands for TPS, the yellow for COMPASS and the blue is the dose difference. 
to see how well both data matched.

\subsubsection{COMPASS Absolute Dose}

Absolute point dose were also extracted in COMPASS (computed and reconstructed) for low and high dose region. These were used to determine confidence limit of point dose determination with COMPASS with respect to chamber measurement and planned dose. The results are presented in Tables 1-4. It shows that COMPASS computed and reconstructed doses are in good confidence limit with TPS dose in high dose region and have an acceptable one in the low dose region (Maximum 6\%).

\subsubsection{Planar dose Distribution}

The measurements were made with the MatriXX and calculated on the TG-119's phantom CT with COMPASS. Planar dose distributions in COMPASS (reconstructed and computed) were extracted in the central plane and also above and below the central plane for the respective cases. The analysis was done using gamma criteria $3 \% / 3 \mathrm{~mm}$ and a threshold of $10 \%$; the percent of point recorded have a gamma lowest of equal to one. Table 5(a) and Table 5(b) show the 2D gamma analysis of dose distributions at the recommended points obtained receptively by COMPASS computed (5a) and COMPASS reconstructed (5b).

Table 1. Compass computed (CC) versus ionization chamber (IC) absolute dose with its confidence limit. (The confidence limit is defined as the sum of the average deviation and 1.96 time of the standard deviation. 1.96 means that $5 \%$ of the individual measurement may exceed the individual limit).

\begin{tabular}{|c|c|c|c|c|c|c|}
\hline Test & $\begin{array}{c}\text { Prescribed } \\
\text { Dose/fraction }\end{array}$ & Location & CC dose & IC dose & $\begin{array}{c}\text { High dose } \\
\text { region } \\
\text { (CC-IC)/Presc }\end{array}$ & $\begin{array}{l}\text { Low dose } \\
\text { region } \\
\text { C-IC)/Presc }\end{array}$ \\
\hline \multirow{3}{*}{ Multi Target } & \multirow{3}{*}{1.8} & isocenter & 1.799 & 1.802 & -0.001 & \\
\hline & & $4 \mathrm{~cm}$ superior & 1.12 & 1.177 & & -0.031 \\
\hline & & $4 \mathrm{~cm}$ inferior & 0.532 & 0.533 & & -0.0006 \\
\hline \multirow{2}{*}{ Prostate } & & Isocenter & 2.034 & 2.021 & 0.006 & \\
\hline & 2 & $2.5 \mathrm{~cm}$ posterior & 1.271 & 1.265 & & 0.003 \\
\hline \multirow{2}{*}{ Head/Neck } & \multirow{2}{*}{2} & isocenter & 2.029 & 1.994 & 0.017 & \\
\hline & & $4 \mathrm{~cm}$ posterior & 1.227 & 1.265 & & -0.019 \\
\hline \multirow{2}{*}{ CShape (easy) } & \multirow{2}{*}{2} & isocenter & 0.583 & 0.533 & & 0.025 \\
\hline & & $2.5 \mathrm{~cm}$ anterior & 2.034 & 1.946 & 0.004 & \\
\hline \multirow{5}{*}{ CShape (hard) } & & isocenter & 0.429 & 0.445 & & -0.008 \\
\hline & 2 & $2.5 \mathrm{~cm}$ anterior & 1.996 & 2.023 & -0.013 & \\
\hline & & \multicolumn{3}{|c|}{ Mean } & 0.010 & -0.005 \\
\hline & & \multicolumn{3}{|c|}{ Standard deviation } & 0.021 & 0.019 \\
\hline & & \multicolumn{3}{|c|}{ Confidence limit $=\mid$ mean $\mid+1.96 \sigma$} & 0.053 & 0.043 \\
\hline
\end{tabular}


Table 2. Compass computed (CC) absolute dose with its confidence limit. (The confidence limit is defined as the sum of the average deviation and 1.96 time of the standard deviation. 1.96 means that $5 \%$ of the individual measurement may exceed the individual limit). IC stands for ionization chamber point dose measurement.

\begin{tabular}{ccccccc}
\hline Test & $\begin{array}{c}\text { Prescribed } \\
\text { Dose/fraction }\end{array}$ & Location & CC dose & Planned dose & $\begin{array}{c}\text { High dose } \\
\text { region } \\
\text { (CC-plan)/presc }\end{array}$ & $\begin{array}{c}\text { Low dose } \\
\text { region }\end{array}$ \\
(CC-plan)/presc
\end{tabular}

Table 3. Compass Reconstructed (CR) versus ionization chamber (IC) absolute dose with its confidence limit. (The confidence limit is defined as the sum of the average deviation and 1.96 time of the standard deviation. 1.96 means that $5 \%$ of the individual measurement may exceed the individual limit).

\begin{tabular}{|c|c|c|c|c|c|c|}
\hline Test & $\begin{array}{c}\text { Prescribed } \\
\text { Dose/fraction }\end{array}$ & Location & CR dose & IC dose & $\begin{array}{c}\text { High dose } \\
\text { region } \\
\text { (CR-IC)/Presc }\end{array}$ & $\begin{array}{c}\text { Low dose } \\
\text { region } \\
\text { (CR-IC)/Presc }\end{array}$ \\
\hline \multirow{3}{*}{ MultiTarget } & \multirow{3}{*}{1.8} & isocenter & 1.788 & 1.802 & -0.007 & \\
\hline & & $4 \mathrm{~cm}$ superior & 1.160 & 1.177 & & -0.009 \\
\hline & & $4 \mathrm{~cm}$ inferior & 0.537 & 0.5331 & & 0.002 \\
\hline \multirow{2}{*}{ Prostate } & \multirow{2}{*}{2} & Isocenter & 2.019 & 2.021 & -0.001 & \\
\hline & & $2.5 \mathrm{~cm}$ posterior & 1.292 & 1.265 & & 0.013 \\
\hline \multirow{2}{*}{ Head/Neck } & \multirow{2}{*}{2} & isocenter & 2.015 & 1.994 & 0.010 & \\
\hline & & $4 \mathrm{~cm}$ posterior & 1.227 & 1.265 & & -0.019 \\
\hline \multirow{2}{*}{ CShape (easy) } & \multirow{2}{*}{2} & isocenter & 0.622 & 0.533 & & 0.044 \\
\hline & & $2.5 \mathrm{~cm}$ anterior & 2.055 & 1.946 & 0.054 & \\
\hline \multirow{5}{*}{ CShape (hard) } & & isocenter & 0.454 & 0.445 & & 0.004 \\
\hline & & $2.5 \mathrm{~cm}$ anterior & 2.007 & 2.023 & -0.008 & \\
\hline & & \multicolumn{3}{|c|}{ Mean } & 0.009 & 0.006 \\
\hline & & \multicolumn{3}{|c|}{ Standard deviation } & 0.026 & 0.021 \\
\hline & & \multicolumn{3}{|c|}{ Confidence limit $=\mid$ mean $\mid+1.96 \sigma$} & 0.060 & 0.049 \\
\hline
\end{tabular}


Table 4. Compass computed (CR) absolute dose with its confidence limit. (The confidence limit is defined as the sum of the average deviation and 1.96 time of the standard deviation. 1.96 means that $5 \%$ of the individual measurement may exceed the individual limit). IC stands for ionization chamber point dose measurement.

\begin{tabular}{|c|c|c|c|c|c|c|}
\hline Test & $\begin{array}{c}\text { Prescribed } \\
\text { Dose/fraction }\end{array}$ & Location & CR point dose & Planned dose & $\begin{array}{c}\text { High dose } \\
\text { region } \\
\text { (CR-plan)/presc }\end{array}$ & $\begin{array}{c}\text { Low dose } \\
\text { region } \\
\text { (CR-plan)/presc }\end{array}$ \\
\hline \multirow{3}{*}{ MultiTarget } & \multirow{3}{*}{1.8} & isocenter & 1.788 & 1.806 & -0.010 & \\
\hline & & $4 \mathrm{~cm}$ superior & 1.160 & 1.210 & & -0.027 \\
\hline & & $4 \mathrm{~cm}$ inferior & 0.537 & 0.597 & & -0.033 \\
\hline \multirow{2}{*}{ Prostate } & \multirow{2}{*}{2} & Isocenter & 2.019 & 2.016 & 0.001 & \\
\hline & & $2.5 \mathrm{~cm}$ posterior & 1.292 & 1.260 & & 0.016 \\
\hline \multirow{2}{*}{ Head/Neck } & \multirow{2}{*}{2} & isocenter & 2.015 & 2.024 & -0.004 & \\
\hline & & $4 \mathrm{~cm}$ posterior & 1.227 & 1.268 & & -0.020 \\
\hline \multirow{2}{*}{ CShape (easy) } & \multirow{2}{*}{2} & isocenter & 0.622 & 0.616 & & 0.003 \\
\hline & & $2.5 \mathrm{~cm}$ anterior & 2.055 & 2.043 & 0.006 & \\
\hline \multirow{5}{*}{ CShape (hard) } & & isocenter & 0.454 & 0.459 & & -0.002 \\
\hline & 2 & $2.5 \mathrm{~cm}$ anterior & 2.007 & 2.016 & -0.004 & \\
\hline & & \multicolumn{3}{|c|}{ Mean } & -0.002 & -0.010 \\
\hline & & \multicolumn{3}{|c|}{ Standard deviation } & 0.006 & 0.019 \\
\hline & & \multicolumn{3}{|c|}{ Confidence limit $=\mid$ mean $\mid+1.96 \sigma$} & 0.014 & 0.048 \\
\hline
\end{tabular}

Table 5. Gamma analysis of planar dose distributions in the central and others planes; (a) is the planes obtain with COMPASS computed dose and (b) is the planes of the reconstructed dose.

(a)

\begin{tabular}{|c|c|c|}
\hline Test & Plane & $\%$ gamma pass \\
\hline MultiTarget & isocenter & 99.09 \\
\hline \multirow[t]{2}{*}{ Prostate } & isocenter & 100 \\
\hline & $2.5 \mathrm{~cm}$ posterior & 98.72 \\
\hline \multirow[t]{2}{*}{ Head/Neck } & isocenter & 95.64 \\
\hline & $4.0 \mathrm{~cm}$ posterior & 95.95 \\
\hline \multirow[t]{2}{*}{ CShape (easy) } & isocenter & 99.76 \\
\hline & $2.5 \mathrm{~cm}$ anterior & 99.96 \\
\hline \multirow[t]{3}{*}{ CShape (hard) } & isocenter & 99.36 \\
\hline & $2.5 \mathrm{~cm}$ anterior & 99.79 \\
\hline & Mean & 98.69 \\
\hline \multicolumn{2}{|c|}{ Standard deviation } & 1.69 \\
\hline \multicolumn{2}{|c|}{ Confidence limit $=\mid$ mean $\mid+1.96 \sigma$} & 4.63 \\
\hline
\end{tabular}


(b)

\begin{tabular}{|c|c|c|}
\hline Test & Plane & $\%$ gamma pass \\
\hline MultiTarget & isocenter & 99.37 \\
\hline \multirow[t]{2}{*}{ Prostate } & isocenter & 100 \\
\hline & $2.5 \mathrm{~cm}$ posterior & 99.6 \\
\hline \multirow[t]{2}{*}{ Head/Neck } & isocenter & 99.07 \\
\hline & $4.0 \mathrm{~cm}$ posterior & 95.41 \\
\hline \multirow[t]{2}{*}{ CShape (easy) } & isocenter & 99.91 \\
\hline & $2.5 \mathrm{~cm}$ anterior & 99.49 \\
\hline \multirow[t]{3}{*}{ CShape (hard) } & isocenter & 99.96 \\
\hline & $2.5 \mathrm{~cm}$ anterior & 99.78 \\
\hline & Mean & 99.17 \\
\hline \multicolumn{2}{|c|}{ Standard deviation } & 1.44 \\
\hline \multicolumn{2}{|c|}{ Confidence limit $=\mid$ mean $\mid+1.96 \sigma$} & 3.65 \\
\hline
\end{tabular}

Table 6. Prostate and head and neck cases: COMPASS computed (CC) and reconstructed (CR) for a $2 \% / 2 \mathrm{~mm}$ criteria, global normalization and $10 \%$ threshold. The passing rate is the percentage of points for gamma lowest or equal to 1 .

\begin{tabular}{|c|c|c|c|c|c|c|c|c|c|c|c|c|}
\hline & \multicolumn{6}{|c|}{$\mathrm{CC}$} & \multicolumn{6}{|c|}{ CR } \\
\hline & \multicolumn{2}{|c|}{ Axial } & \multicolumn{2}{|c|}{ Coronal } & \multicolumn{2}{|c|}{ Sagittal } & \multicolumn{2}{|c|}{ Axial } & \multicolumn{2}{|c|}{ Coronal } & \multicolumn{2}{|c|}{ Sagittal } \\
\hline & $\gamma$ & SD & $\gamma$ & SD & $\gamma$ & SD & $\gamma$ & SD & $\gamma$ & SD & $\gamma$ & SD \\
\hline P1 & 98.04 & 0.24 & 93.64 & 0.36 & 94.25 & 0.33 & 95.91 & 0.31 & 91.75 & 0.34 & 90.07 & 0.35 \\
\hline P2 & 99.5 & 0.20 & 98.66 & 0.24 & 99.23 & 0.22 & 90.14 & 0.34 & 90.51 & 0.39 & 93.61 & 0.32 \\
\hline P3 & 100 & 0.12 & 96.77 & 0.29 & 99.06 & 0.21 & 93.55 & 0.32 & 97.29 & 0.28 & 98.48 & 0.24 \\
\hline P4 & 99.79 & 0.18 & 98.98 & 0.22 & 99.5 & 0.20 & 87.51 & 0.33 & 96.06 & 0.29 & 95.21 & 0.30 \\
\hline P5 & 99.45 & 0.17 & 99.93 & 0.15 & 99.71 & 0.19 & 96.04 & 0.26 & 99.24 & 0.23 & 96.86 & 0.28 \\
\hline HN1 & 93.73 & 0.35 & 94.66 & 0.35 & 97.67 & 0.27 & 95.65 & 0.34 & 93.77 & 0.38 & 97.73 & 0.34 \\
\hline HN2 & 97.11 & 0.28 & 96.57 & 0.29 & 92.57 & 0.38 & 96.41 & 0.30 & 89.29 & 0.42 & 85.08 & 0.48 \\
\hline HN3 & 90.57 & 0.41 & 94.02 & 0.40 & 90.1 & 0.46 & 92.23 & 0.37 & 96.01 & 0.33 & 93.23 & 0.38 \\
\hline HN4 & 98.99 & 0.24 & 98.04 & 0.24 & 97.08 & 0.27 & 88.63 & 0.22 & 99.92 & 0.18 & 97.18 & 0.30 \\
\hline HN5 & 90.177 & 0.40 & 93.87 & 0.33 & 96.98 & 0.28 & 74.64 & 0.55 & 76.82 & 0.46 & 84.22 & 0.37 \\
\hline Mean & 96.73 & 0.25 & 96.51 & 0.28 & 96.61 & 0.28 & 91.07 & 0.33 & 93.06 & 0.33 & 93.16 & 0.33 \\
\hline
\end{tabular}

\subsection{Validation with Real Patients}

For ten real patients (head and neck and prostate), 2D gamma was extracted in the plan of the isocenter for the three directions (axial, coronal and sagittal). Gamma passing rate for the planes have $2 \% / 2 \mathrm{~mm}$ and $3 \% / 3 \mathrm{~mm}$ tolerance are represented on Table 6 and Table 7.

With COMPASS DVH tool, the differences for some dosimetric parameters were calculated for our group of ten patients. Table 8 and Table 9 show these differences in target volumes and normal tissues for ten patients (head and 
Table 7. Prostate and head and neck cases: COMPASS computed (CC) and reconstructed (CR) for a 3\%/3mm criteria, global normalization and $10 \%$ threshold. The passing rate is the percentage of points for gamma lowest or equal to 1 .

\begin{tabular}{|c|c|c|c|c|c|c|c|c|c|c|c|c|}
\hline & \multicolumn{6}{|c|}{$\mathrm{CC}$} & \multicolumn{6}{|c|}{ CR } \\
\hline & \multicolumn{2}{|c|}{ Axial } & \multicolumn{2}{|c|}{ Coronal } & \multicolumn{2}{|c|}{ Sagittal } & \multicolumn{2}{|c|}{ Axial } & \multicolumn{2}{|c|}{ Coronal } & \multicolumn{2}{|c|}{ Sagittal } \\
\hline & $\gamma$ & SD & $\gamma$ & SD & $\gamma$ & SD & $\gamma$ & SD & $\gamma$ & SD & $\gamma$ & SD \\
\hline $\mathrm{P} 1$ & 99.46 & 0.20 & 99.77 & 0.16 & 99.36 & 0.22 & 99.48 & 0.24 & 100 & 0.17 & 99.27 & 0.24 \\
\hline $\mathrm{P} 2$ & 99.98 & 0.13 & 99.9 & 0.16 & 100 & 0.15 & 99.21 & 0.22 & 97.25 & 0.26 & 99.81 & 0.21 \\
\hline P3 & 100 & 0.08 & 99.26 & 0.18 & 99.91 & 0.14 & 99.21 & 0.21 & 99.55 & 0.20 & 99.86 & 0.16 \\
\hline $\mathrm{P} 4$ & 99.98 & 0.12 & 100 & 0.13 & 100 & 0.13 & 99.64 & 0.22 & 98.9 & 0.22 & 99.84 & 0.20 \\
\hline P5 & 100 & 0.11 & 100 & 0.10 & 100 & 0.13 & 100 & 0.18 & 100 & 0.15 & 100 & 0.18 \\
\hline HN1 & 98.65 & 0.25 & 98.33 & 0.23 & 99.35 & 0.21 & 99.15 & 0.23 & 98.26 & 0.25 & 99.82 & 0.19 \\
\hline HN2 & 99.83 & 0.19 & 99.46 & 0.23 & 98.01 & 0.26 & 99.86 & 0.21 & 97.28 & 0.28 & 95.35 & 0.32 \\
\hline HN3 & 97.49 & 0.28 & 96.96 & 0.27 & 97.37 & 0.26 & 98.48 & 0.25 & 98.75 & 0.22 & 97.82 & 0.25 \\
\hline HN4 & 100 & 0.16 & 100 & 0.16 & 99.75 & 0.18 & 100 & 0.15 & 100 & 0.12 & 99.63 & 0.20 \\
\hline HN5 & 98.16 & 0.27 & 99.13 & 0.22 & 99.83 & 0.19 & 92.56 & 0.37 & 95.13 & 0.31 & 99.44 & 0.25 \\
\hline Mean & 99.35 & 0.17 & 99.28 & 0.18 & 99.35 & 0.18 & 98.75 & 0.22 & 98.51 & 0.21 & 99.08 & 0.22 \\
\hline
\end{tabular}

Table 8. COMPASS computes (CC) and reconstructed (CR) differences for dosimetric parameters (PTV and normal tissues) for five VMAT plans of prostate for three dose levels.

\begin{tabular}{|c|c|c|c|c|c|c|c|c|c|c|c|c|c|}
\hline & & \multicolumn{12}{|c|}{ Differences (\%) } \\
\hline & & \multicolumn{6}{|c|}{$\mathrm{CC}$} & \multicolumn{6}{|c|}{ CR } \\
\hline & & P1 & P2 & P3 & P4 & P5 & Mean & P1 & P2 & $\mathrm{P} 3$ & P4 & P5 & Mean \\
\hline \multirow{4}{*}{ PTV1 } & D98 & 0.23 & 0.29 & -0.14 & 0.35 & 0.45 & 0.23 & -0.69 & -1.3 & -0.5 & 1.29 & -0.26 & -0.29 \\
\hline & D95 & 1.3 & 0.3 & 0.14 & 0.63 & 0.43 & 0.56 & 0.35 & -1.23 & -0.61 & 0.62 & -0.39 & -0.25 \\
\hline & D50 & 1.31 & 0.31 & 0.81 & 0.58 & 0.33 & 0.66 & -0.21 & -1.44 & -0.12 & 0.99 & -0.64 & -0.28 \\
\hline & $\mathrm{D} 2$ & 1.28 & 0.79 & 1.56 & 0.48 & -0.42 & 0.73 & -0.26 & -1.18 & 0.45 & 0.49 & -1.05 & -0.31 \\
\hline PTV2 & D95 & 0.04 & 0.14 & 0.07 & -0.69 & 0.75 & 0.06 & -0.78 & -0.35 & -0.6 & -0.92 & 0.05 & -0.52 \\
\hline PTV3 & D95 & -0.29 & -0.86 & 0.32 & -0.85 & -0.78 & -049 & -1.23 & -0.58 & -0.32 & -0.35 & -1.85 & -0.86 \\
\hline \multirow{4}{*}{ Rectum } & V50 & -0.12 & -0.03 & -1.32 & -0.72 & -0.87 & -0.61 & -1.19 & -1.39 & -1.28 & -1.62 & -1.27 & -1.35 \\
\hline & V60 & 0.42 & 1.21 & -0.44 & -0.20 & -0.01 & 0.19 & -0.64 & -0.47 & -0.44 & -0.87 & -0.87 & -0.65 \\
\hline & V70 & 0.65 & 1.13 & 0.2 & 0.35 & 0.17 & 0.05 & -0.07 & -0.74 & -0.80 & -1.96 & -0.13 & -0.74 \\
\hline & V74 & 1.53 & 0 & 0.17 & 0.09 & 0.02 & 0.36 & 0 & 0 & -0.28 & -0.17 & -0.01 & -0.09 \\
\hline \multirow{3}{*}{ Bladder } & V60 & -0.27 & -0.08 & -0.06 & -0.05 & -0.09 & -0.11 & -0.95 & -0.10 & 0.01 & -0.01 & -0.29 & -0.26 \\
\hline & V70 & 1.06 & 0.13 & -0.52 & 0.08 & 0.07 & 0.16 & 0.13 & -0.44 & -0.29 & 0.01 & -0.45 & -0.20 \\
\hline & V74 & 0.36 & 0.67 & -0.23 & 2.05 & -0.17 & 0.53 & -0.03 & -0.16 & 0.15 & 0.25 & -1.52 & -0.26 \\
\hline $\mathrm{R}$ fem & D10 & 0.83 & -0.47 & 0.35 & -0.29 & 0.19 & 0.12 & -2.22 & -2.02 & -3.47 & -2.02 & -1.58 & -2.26 \\
\hline L fem & D10 & -0.03 & -0.2 & 0.77 & 0.98 & 0.2 & 0.34 & -1.97 & -1.17 & -1.02 & -2.16 & -2.26 & -1.71 \\
\hline
\end{tabular}


Table 9. COMPASS computes (CC) and reconstructed (CR) differences for dosimetric parameters (PTV and normal tissues) for five VMAT plans of head and neck for three dose levels. The maximal dose (Dmax) of cord in COMPASS is assumed to be the dose at the volume $0.01 \%$.

\begin{tabular}{|c|c|c|c|c|c|c|c|c|c|c|c|c|c|}
\hline & & \multicolumn{12}{|c|}{ Difference (\%) } \\
\hline & & \multicolumn{6}{|c|}{$\mathrm{CC}$} & \multicolumn{6}{|c|}{$\mathrm{CR}$} \\
\hline & & HN1 & HN2 & HN3 & HN4 & HN5 & Mean & HN1 & HN2 & HN3 & HN4 & HN5 & Mean \\
\hline \multirow{4}{*}{ PTV1 } & D98 & 0.86 & 0.69 & 1.3 & 0.52 & -0.33 & 0.60 & 1.84 & 0.03 & 0.21 & -0.12 & -1.15 & 0.16 \\
\hline & D95 & -0.25 & 0.75 & 1.37 & 0.63 & -0.36 & 0.42 & 0.8 & 0.12 & 0.2 & -0.07 & -1.16 & -0.02 \\
\hline & D50 & -0.19 & 1.11 & 2.87 & 0.7 & -0.07 & 0.88 & 0.15 & 0.4 & 1.58 & 0.03 & -1 & 0.23 \\
\hline & $\mathrm{D} 2$ & 0.27 & 1.2 & 2.91 & 0.71 & -0.54 & 0.91 & 0.03 & 0.44 & 1.29 & 0.42 & -0.26 & 0.38 \\
\hline PTV2 & D95 & -0.40 & 0.26 & 0.47 & 0.08 & 0.63 & 0.36 & -0.75 & -0.68 & -1.02 & -0.27 & -1.18 & -0.78 \\
\hline PTV3 & D95 & -0.54 & 0.54 & 0.05 & 0.65 & -0.13 & 0.11 & -1.06 & -1.05 & -1.66 & -0.74 & -1.69 & -1.24 \\
\hline Cord & Dmax & -2.28 & -2.16 & -1.23 & -2.74 & -6.01 & -2.88 & -3.92 & -2.15 & -3.84 & -2.73 & -5.38 & -3.30 \\
\hline R Parot & D50 & -0.79 & -4.02 & 0.27 & 0.33 & -4.85 & -1.81 & -1.58 & -12.06 & -0.26 & 1.63 & -5.91 & -3.63 \\
\hline L Parot & D50 & 2.81 & 1.69 & 11.41 & 5.84 & -4.17 & 3.51 & -1.23 & -3.57 & 5.37 & 3.44 & -1.42 & 0.51 \\
\hline
\end{tabular}

neck and prostate).

For Prostate case, PTVs ICRU dose points, the mean absolute difference dose value is lower than $1 \%$ for both COMPASS reconstructed and computed dose. Reconstructed dose seems to be lower than the TPS calculated dose. For OARs, the mean difference on tolerance dose is lower than $1 \%$ for CC and $2.5 \%$ for CR. COMPASS reconstructed dose for OARs are in all the case lower than TPS calculated one.

For head and neck case, PTVs ICRU dose points, the mean absolute difference dose value is lower than $1 \%$ for both COMPASS reconstructed and computed dose. Reconstructed dose seems to be lower than the TPS calculated dose. Parotids dose determination has a strong dependence to the beam modeling beyond the buildup region. Maximum dose determination in COMPASS is defined as the dose in a $0.01 \%$ volume for spinal cord.

\subsection{Plans with Intentional Errors}

For a Prostate and a head and neck plan, we included some intentional errors to evaluate their influences on plan quality. Using the normal plan as reference, we extracted the 3D global gamma, the average gamma on organs and the dose difference for PTV and OAR. The following Table 10 and Table 11 summarized these values and show the influence of each error on PTV and OARs.

\section{Discussion}

The geometric resolution is the main limitation of $2 \mathrm{D}$ arrays detectors. This limitation is not observed in measurement-base dose reconstruction performed by COMPASS because of the inherent correction of the system.

The AAPM TG-119 test analysis planar dose distribution for low and high 
Table 10. COMPASS reconstructed dose for prostate with intentional dose, MLC, Gantry and collimator errors. The plan with no error is take as reference to evaluate the gamma and dose difference.

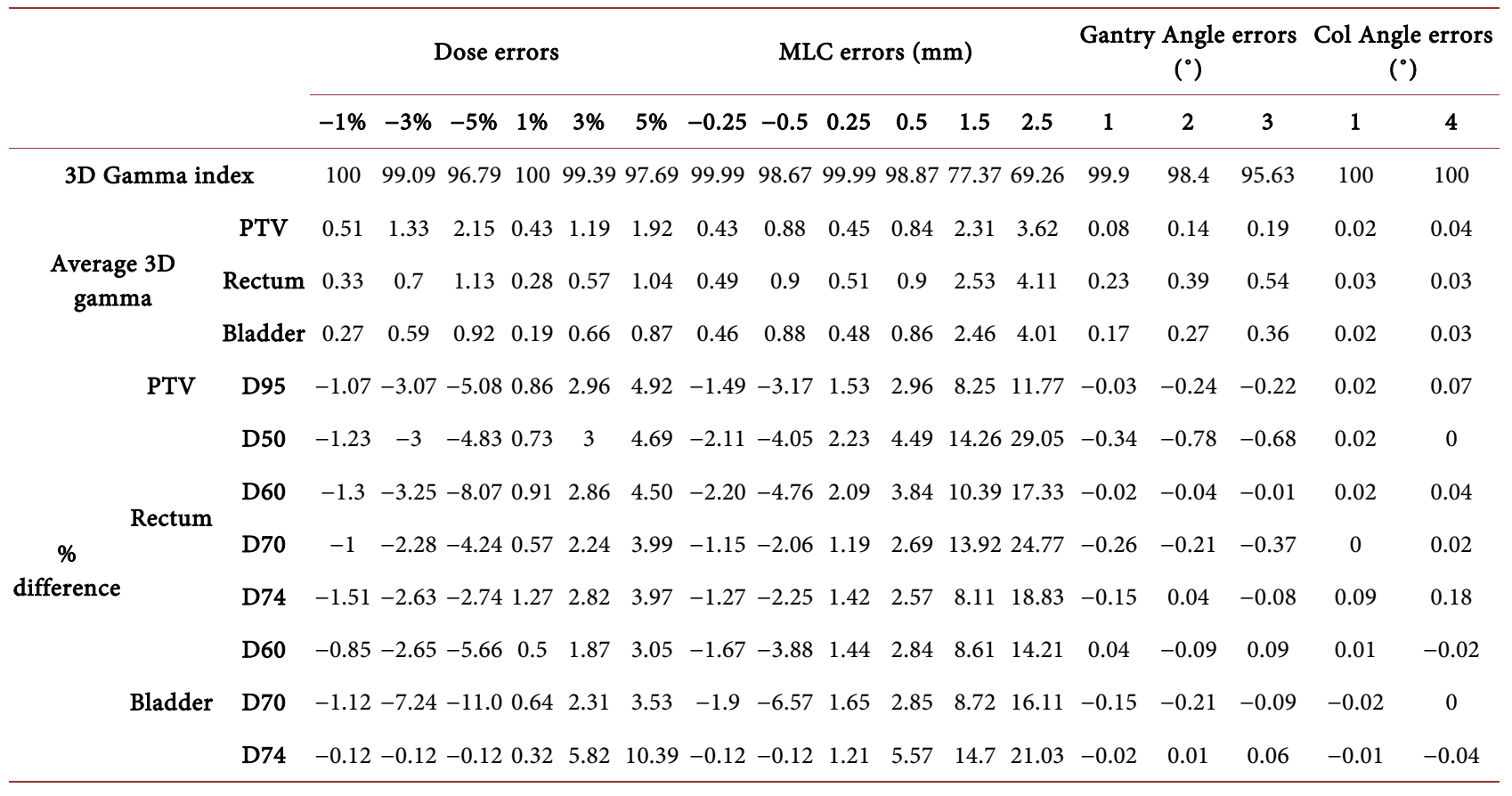

Table 11. COMPASS reconstructed dose for prostate with intentional dose, MLC, Gantry and collimator errors. The plan with no error is take as reference to evaluate the gamma and dose difference.

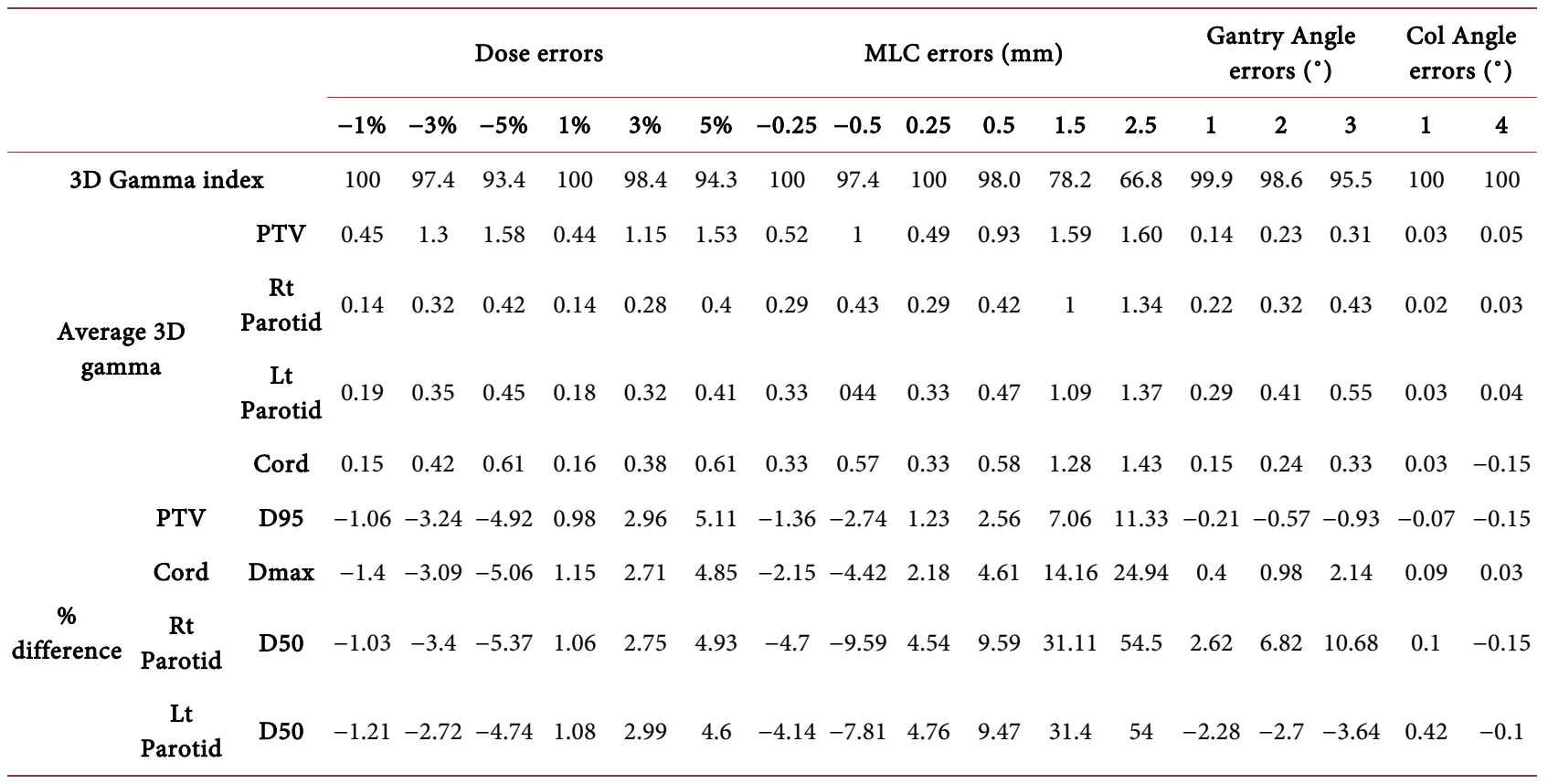

doses shows that our results average, the standard deviation and the confidence limits are comparable to the one of Ezzel et al. [13]. Improving COMPASS beam model of low dose can improve measurements for low dose. The low dose components of energy spectrum COMPASS beam model could be also improved to facilitate this modeling and have better score for dose determination in low dose 
region. Gamma passing rates with global normalization for CC and CR were above the one for the TG-119 for composite dose gamma analysis.

According to Nelms et al. [16], it's important to perform analysis with more strict criteria than the traditional one. For our COMPASS computed and reconstructed validation, we used for 2D dose distributions the gamma analysis of $2 \% /$ $2 \mathrm{~mm}$ and $3 \% / 3 \mathrm{~mm}$ criteria in the three planes. COMPASS allows for $2 \mathrm{D}$ gamma analysis only a global normalization. That was one of the limitations of the present study. The local 3D gamma analysis at $2 \% / 2 \mathrm{~mm}$ highlighted some failing points mainly located the low dose regions and in the skin. A $3 \% / 3 \mathrm{~mm}$ criteria reduces these falling points. The Dose Volume comparison between TPS and COMPASS (CC and CR) shows very good agreement for both target and OARs. Table 10 and Table 11 show that intentional errors on collimator have minor effect on the dose difference in the patient. Plans are more sensitive to MLC dose errors. Gantry errors induced some difference in dose but not at the same level as MLC.

According to gamma analysis, some MLC errors values could have still been validated. But if we take into consideration the dose difference on DVH, those plans would have not been accepted. That is also a proof that gamma metric has a limitation for a rigorous pretreatment plan validation. COMPASS should deeply change the way physicist commission linac for VMAT treatment. This study was done with a $2 \mathrm{~mm}$ isotropic dose grid resolution that can be very time consuming for COMPASS application (As example, a prostate case, respectively 5 and 3 minutes for 2 and $3 \mathrm{~mm}$ grid size). For clinical implementation, a large grid ( 3 $\mathrm{mm}$ for example) could be less time consuming while keeping the same level of accuracy.

\section{Conclusions}

Unfortunately, point dose measurements and 2D gamma analysis don't give any information on the coverage of PTV or in dose on OAR's. Thus COMPASS with his $3 \mathrm{D}$ analysis tool has a real added value on the clinical value of pretreatment QA process. The $3 \mathrm{D}$ dose reconstruction on patient $\mathrm{CT}$ gives the possibility to obtain planar and volumetric information. The use of a CC algorithm in COMPASS allows the comparison of our TPS dose calculation (Pinnacle) to COMPASS dose computed.

The quantification of the volumetric dose distribution in plans is the weakness of traditional QA tools. The dose reconstruction on CT by COMPASS brought a $3 \mathrm{D}$ dose distribution and the possibility to explore it in many ways as $3 \mathrm{D}$ gamma, DVH etc. Computed dose in COMPASS by a CC algorithm dose engine enables to make a second check of dose calculation by the TPS. Although the post-processing of measurements can be time consuming with COMPASS, it definitely changes the pretreatment quality assurance workflow. Based on the results of this study, we concluded that COMPASS computed and reconstructed doses are in good agreement according to dosimetric benchmarks. The system 
can clinically be implemented with a good level of confidence.

\section{Acknowledgements}

The authors are grateful to the Abdus Salam ICTP for its support through the OEA-AF-12 project at CEPAMOQ.

\section{Conflicts of Interest}

The authors declare no conflicts of interest regarding the publication of this paper.

\section{References}

[1] Otto, K. (2008) Volumetric Modulated Arc Therapy: IMRT in a Single Gantry Arc. Medical Physics, 35, 310-317. https://doi.org/10.1118/1.2818738

[2] Bedford, J., Lee, Y., Wai, P., South, C. and Warrington, A. (2009) Evaluation of the Delta4 phantom for IMRT and VMAT Verification. Physics in Medicine \& Biology, 54, N167-N176. https://doi.org/10.1088/0031-9155/54/9/N04

[3] Bouchard, H. and Seuntjens, J. (2004) Ionization Chamber-Based Reference Dosimetry of Intensity Modulated Radiation Beams. Medical Physics, 31, 2454-2465. https://doi.org/10.1118/1.1781333

[4] Boggula, R., Birkner, M., Lohr, F., Steil, V., Wenz, F. and Wertz, H. (2011) Evaluation of a 2D Detector Array for Patient-Specific VMAT QA with Different Setups. Physics in Medicine \& Biology, 56, 7163-7177. https://doi.org/10.1088/0031-9155/56/22/011

[5] Dobler, B., Streck, N., Klein, E., Loeschel, R., Haerti, P. and Koelbl, O. (2010) Hybrid Plan Verification for Intensity-Moduated Radiation Therapy (IMRT) Using the 2D Ionization Chamber Array I'mRT MatriXX-A Feasibility Study. Physics in Medicine \& Biology, 55, N39-N55. https://doi.org/10.1088/0031-9155/55/2/N02

[6] Boggula, R., Lorenz, F., Mueller, L., Birkner, M., Wertz, H., Stieler, F., et al. (2010) Experimental Validation of a Commercial 3D Dose Verification System for Intensity-Modulated Arc Therapies. Physics in Medicine \& Biology, 55, 5619-5633. https://doi.org/10.1088/0031-9155/55/19/001

[7] Swamy, S.T., Anuradha, C., Kathirvel, M., Arun, G. and Subramanian, S. (2014) Pretreatment Quality Assurance of Volumetric Modulated Arc Therapy on Patient CT Scan Using Indirect 3D Dosimetry System. International Journal of Cancer Therapy and Oncology, 2, Article ID: 020416. http://doi.org/10.14319/ijcto.0204.16

[8] Clemente-Gutiérrez, F. and Pérez-Vara, C. (2015) Dosimetric Validation and Clinical Implementation of Two 3D Dose Verification Systems for Quality Assurance in Volumetric-Modulated arc Therapy Techniques. Journal of Applied Clinical Medical Physics, 16, 198-217. https://doi.org/10.1120/jacmp.v16i2.5190

[9] Stakchall, G., Steadham, R.E., Popple, R.A., Ahmad, S. and Rosen, I.I. (2000) Beam Commissioning Methodology for a Three-Dimensional Convolution/Superposition Photon Dose Algorithm. Journal of Applied Clinical Medical Physics, 1, 8-27. https://doi.org/10.1120/jacmp.v1i1.2651

[10] Cosgrove, V.P., Thomas, M.D.R., Weston, S.J., Thompson, M.G., Reynaert, N., Evans, C.J., et al. (2009) Physical Characterization of a New Design of an ELekta Radiation Head with Integrated 160-Leaf Multileaf Collimator. International Journal of Radiation Oncology, Biology, Physics, 75, S722-S723.

https://doi.org/10.1016/j.ijrobp.2009.07.1646 
[11] Bedford, J.L., Childs, P.J., Nordmark Hansen, V., Mosleh-Shirazi, M.A., Verhaegen, F. and Warrington, A.P. (2003) Commissioning and Quality Assurance of the Pinnacle Radiotherapy Treatment Planning System for External Beam Photons. British Journal of Radiology, 76, 163-176. https://doi.org/10.1259/bjr/42085182

[12] Bedford, J.L., Thomas, M.D.R. and Smyth, G. (2013) Beam Modeling and VMAT Performance with the Agility 160-Leaf Multileaf Collimator. Journal of Applied Clinical Medical Physics, 14, 172-185. https://doi.org/10.1120/jacmp.v14i2.4136

[13] Ezzell, G.A., Galvin, J.M., Low, D., Palta, J.R., Rosen, I., Sharpe, M.B., et al. (2003) Guidance Document on Delivery, Treatment Planning, and Clinical Implementation of IMRT: Report of the IMRT Subcommittee of the AAPM Radiation Therapy Committee. Medical Physics, 30, 2089-2115. https://doi.org/10.1118/1.1591194

[14] Ezzell, G., Burmeister, J., Dogan, N., LoSasso, T.J., Mechalakos, J.G., Mihailidis, D. et al. (2009) IMRT Commissioning: Multiple Institution Planning and Dosimetry Comparisons, a Report from AAPM Task Group 119. Medical Physics, 36, 53595373. https://doi.org/10.1118/1.3238104

[15] Cadman, P., McNutt, T.R. and Bzdusek, K. (2005) Validation of Physics Improvements for IMRT with a Commercial Treatment Planning System. Journal of Applied Clinical Medical Physics, 6, 74-86. https://doi.org/10.1120/jacmp.v6i2.2083

[16] Nelms, B., Chan, M., Jarry, G., Lemire, M., Lowden, J., Hampton, C., et al. (2013) Evaluating IMRT and VMAT Dose Accuracy: Practical Examples of Failure to Detect Systematic Errors When Applying a Commonly Used Metric and Action Levels. Medical Physics, 40, Article ID: 111722. https://doi.org/10.1118/1.4826166 